\title{
Genetic Analysis and Targeted Therapy Using Buparlisib and MK2206 in a Patient with Triple Metachronous Cancers of the Kidney, Prostate, and Squamous Cell Carcinoma of the Lung: A Case Report
}

\author{
Tong Zhao' \\ Yuqin $\operatorname{Tian}^{2}$ \\ Xinjia Ding ${ }^{3}$ \\ Lin $\mathrm{Liu}^{4}$ \\ Bowen $\operatorname{Tan}^{5}$ \\ Bin Yang ${ }^{5}$ \\ Jianlin $\mathrm{Wu}^{5}$ \\ Ting Lei ${ }^{6}$ \\ Ruoyu Wang ${ }^{2}$ \\ Yan Ding $\mathbb{D}^{5,7}$
}

'Department of Oncology, The Affiliated Zhongshan Hospital of Dalian University, Dalian, People's Republic of China;

${ }^{2}$ Department of Care Operations, The Affiliated Hospital of Qingdao University, Qingdao, People's Republic of China;

${ }^{3}$ Department of Neurosurgery, The Second Affiliated Hospital of Dalian Medical University, Dalian, People's Republic of China; ${ }^{4} \mathrm{Navy}$ Qingdao Special Care Center, Qingdao, 26607I, People's Republic of China; ${ }^{5}$ The Institute for Translational Medicine, The Affiliated Zhongshan Hospital of Dalian University, Dalian, People's Republic of China; ${ }^{6}$ Department of Thoracic Surgery, The Second Affiliated Hospital of Dalian Medical University, Dalian, People's Republic of China; ${ }^{7}$ Department of Pediatrics, Children's Hospital of Boston, Harvard Medical School, Boston, MA, 02II5, USA

Correspondence: Yan Ding; Ruoyu Wang Email yan.ding@childrens.harvard.edu; wangruoyul963@163.com

\begin{abstract}
Multiple primary cancers (MPC) occurring in the same individual is considered rare but being increasingly recognized owing to the longer cancer survival nowadays. Despite of accumulating experience in diagnosis, effective treatment remains to be problematic in many scenarios. Genetic testing-based targeted therapy could be an invaluable option for both diagnosis and treatment of such patients. Here we present a 74-year-old male with triple primary cancers including kidney, prostate, and lung with metastatic tumor on the costal bones. The patient visited the hospital for persistent cough and hemoptysis, and a diagnosis of squamous cell carcinoma of the left lung was made by bioptic fiberoptic bronchoscopy. A previous history included renal cancer controlled by Sorafenib and prostate cancer controlled by Goserelin. Radiotherapy and platinum-based chemotherapy failed to help the patient and the tumor size increased over a period of 6 months. In order to seek better therapeutical options, we performed targeted sequencing using the cancerous tissues from his lung, kidney, and prostate cancers. Briefly, the results identified VHL, EGFR, PIK3CA, TP53, and AKT1 mutations in lung cancer, AKT1, FGFR2, and TP53 mutations in renal cancer, and FGFR2 mutations in prostate cancer. A combined medication targeting PIK3CA and AKT1 signaling was recommended and the patient was given BKM120 (PIK3CA, Phase III clinical trial) and MK2206 (AKT, phase III clinical trial). Revisit chest CTs after 4 months and 9 months showed a significant shrinkage of tumor size by $40 \%$ and $80 \%$, respectively. Our experience demonstrated a good example that genetic analysis could be valuable to diagnose and precisely treat multiple primary cancers.
\end{abstract}

Keywords: multiple primary cancers, next generation sequencing, targeted therapy, Buparlisib, BKM120, MK2206

\section{Background}

Multiple primary cancers (MPC) occurring in the same individual is rare but being more recognized owing to the prolonged lifespan and improved cancer survival nowadays. ${ }^{1}$ Cancer is a genomic disorder that advancing age ranks the top risk factor, and the lifetime risk for any cancer is estimated as $1 / 2$ to $1 / 3{ }^{2}$ The prevalence of two or more primary tumors in the US population is $0.27 \%$ based on a report in $2007 .{ }^{1}$ Therefore, it becomes increasingly important to accumulate 
the understanding of molecular basis and therapeutical experience in regard to MPC diagnosis and treatment.

However, selection of the best therapeutical options for MPC remains difficult. ${ }^{1,3}$ The specific scenario of MPC varies depending on the cancer type, location, TNM stage and degree of malignancy. Surgery remains first-line but must satisfy appropriate indications. Chemotherapy is commonly needed, but each regimen is specific to treat certain cancers and may be limited to manage multiple cancers. Alternative approaches such as genetic testingguided targeted therapy could be a valuable option with customized designation. Furthermore, an accurate differential diagnosis is important for MPC diagnosis, usually relying on the histopathological methods, and yet genome analysis could be additionally helpful to precisely determine the tumor clonality.

In the present case, a male patient was diagnosed with triple primary cancers of the clear cell renal cell carcinoma, prostate adenocarcinoma, squamous cell carcinoma (SQCC) of the lung and had a history of tumor metastasis on costal bones. There were no surgical indications for the SQCC. Radiotherapy was used but failed soon. The patient was thereafter treated with platinum-based chemotherapy but the tumor continued to grow. Gene testing-guided targeted therapy was employed and a durable response was evidenced.

\section{Case Presentation}

A 74-year-old male first presenting in July 2014 complained with chest pain, persistent dry cough, and intermittent hemoptysis during the past 4 months. Chest CT showed nodular opacity $(1.7 \mathrm{~cm})$ occupying the left main bronchus. A diagnosis of poor-differentiated SQCC (T1NxM0) was subsequently made by flexible bioptic fiberoptic bronchoscopy and immunohistochemistry staining of SQCC biomarkers (Figure 1A-D). The patient had a smoking history of 30 pack-years.

His medical history had many significant aspects, including: 1) a previous diagnosis of primary clear cell renal cell carcinoma (CCRCC), grade 2, of the right kidney in June 2008 (Figure 1E and F). An open surgery observed a $4.0 \mathrm{~cm}$ tumor and radical nephrectomy was performed; 2) multiple metastatic bone tumors (Left: 7th rib, Right: 5th, 10th, 11th ribs). The patient complained of pain in the right rib cage in December 2012 and FDG-PET $\mathrm{CT}$ and chest $\mathrm{CT}$ suspected metastasis. A partial rib resection was performed on the 11 th right rib and pathology observed the presence of clear cells (Figure $1 \mathrm{G}$ and $\mathrm{H}$ ). In
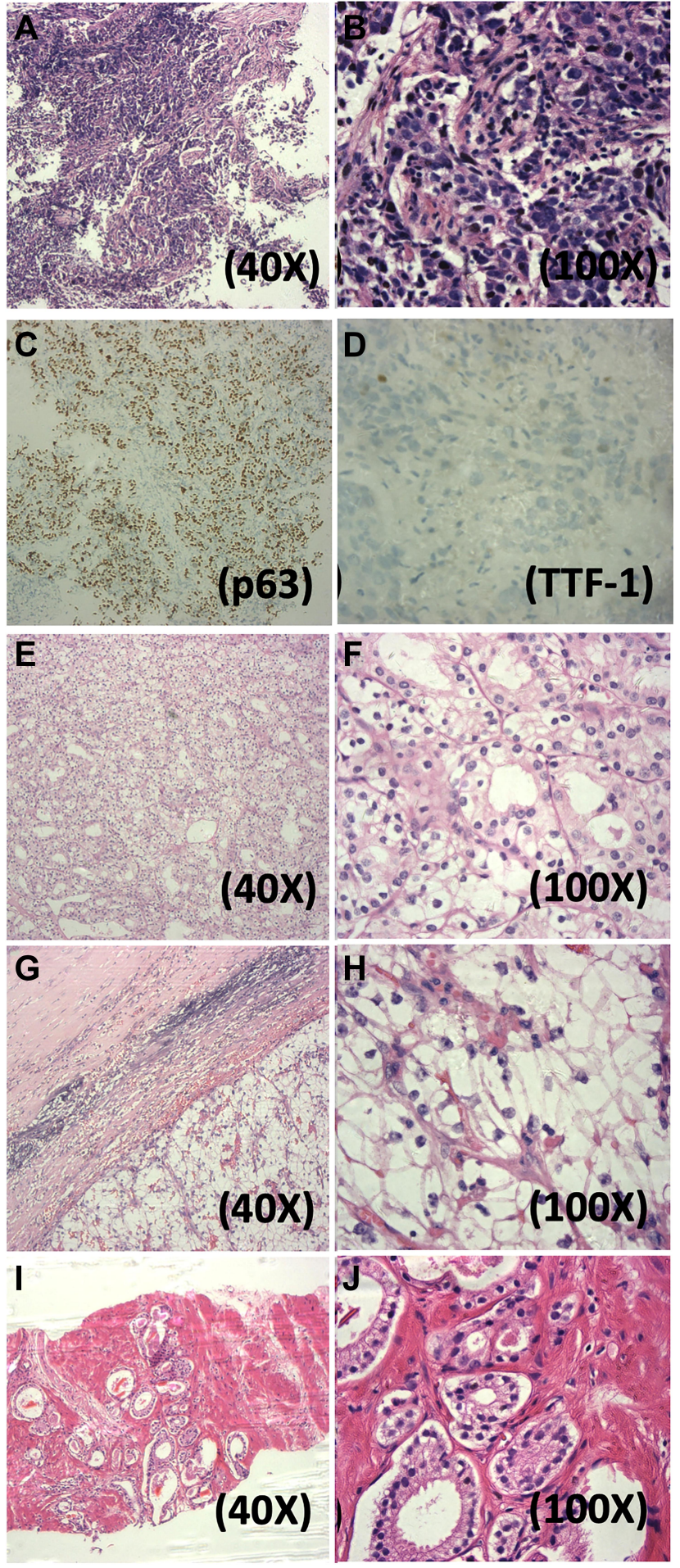

Figure I H\&E staining of triple primary tumors and metastatic bone tumors of the case. (A and B) Primary lung SQCC (40x, 100x); (C and D) Immunohistochemistry staining of p63 and TTFI for SQCC; (E and F) Primary CCRCC (40x, 100x); (G and H) Metastatic bone tumor (40x, 100x); (I and J) Primary prostate adenocarcinoma.

June 2013, bone scintigraphy suspected recurring bone metastasis on the 10th right rib. The patient started to take oral Sorafenib and Zoledronate since June 2013. 3) 
A previous diagnosis of primary prostate adenocarcinoma in January 2014, accidentally identified by prostate specific-antigen (PSA) check-up and needle biopsy (Table S1 and Figure 1I and J). Chemical castration using Goserelin and Bicalutamide decreased the PSA level to under 0.01 $\mathrm{ng} / \mathrm{mL}$. PSA level was thereafter monitored (Table S1) and intermittent Goserelin was administered whenever the PSA level was found rebooted.

The patient's lung SQCC (Figure 2A) was inoperable since a left pneumonectomy was not possible. Palliative radiation therapy as DT:7480CGY/220CGY/34F was initiated. Tachypnea and dyspnea were soon reported and $\mathrm{CT}$ suggested radiation pneumonitis with increased tumor size (Figure 2B and C). Dexamethasone was used to resolve the pneumonitis (October-November 2014, Figure 2C), and radiotherapy was discontinued in November 2014. Next, the patient was treated with platinum-based chemotherapy (etoposide $120 \mathrm{mg} / \mathrm{m}^{2}$, IVGTT plus cisplatin $75 \mathrm{mg} / \mathrm{m}^{2}$, IVGTT or carboplatin $200 \mathrm{mg} /$ $\mathrm{m}^{2}$, IGVTT, q21d/c) for five cycles from November 2014 to March 2015, but the tumor size was found to be significantly increased in February 2015 (Figure 2D and E), after which chemotherapy was maintained for one more cycle and soon abandoned in March 2015. By then, the patient had exhausted all the routine approaches and, at the same time, he had the kidney cancer under SorafenibZoledronate control and unresected prostate cancer under Goserelin control.

To solve the problem, we considered genome-based targeted therapy, which had a proven excellent effect to treat various malignancies including lung adenocarcinoma, colorectal adenocarcinoma, ovarian, and breast cancers. ${ }^{4}$ Targeted sequencing of all four tumor tissues from lung, kidney, prostate, and bone was performed using TruSeq Amplicon Cancer Panel (Illumina) and KnoTruth ${ }^{\mathrm{TM}}$ OncUro-600 panel (JIEN Biotechnologies, China) on the Illumina NextSeq500 next generation sequencing (NGS) platform. The NGS data was analyzed using OncoDecoder $^{\mathrm{TM}}$ (Genomic Future, Inc. USA), The rationale to include all tumor tissues into the NGS analysis was in hope to better strategize the therapeutical plan by understanding the mutational landscapes of all the cancers and to use a minimal number of drugs and to expect manageable toxicity.

The genome sequencing results revealed actionable somatic mutations of several driver genes (Table 1). Lung SQCC was found to have mutations in VHL, PIK3CA, EGFR, HRAS, TP53, BRAF, CDH1, and AKT1. CCRCC harbored mutations in VHL, FGFR2 and AKT1, and TP53 The prostate tumor does not carry any actionable mutation. The metastatic bone tumor of CCRCC origin showed mutations in VHL, FGFR2, and TP53. The VHL, FGFR2, and TP53 mutations were the most common ones, with the VHL mutations in lung, renal and bone tumors, and the FGFR2 mutations in renal and metastatic bone tumors. Several PIK3CA and AKT1 mutations are canonical and had been reported in various cancers according to COSMIC and Clinvar database, including PIK3CA c.3142C $>$ T $\mid$ p. His1048Tyr (COSM249875), AKT1 c.68G>A|p.Arg23Gln (COSM3770600), and c.49G>A|p.Glu17Lys (COSM33765).

Because the patient has exhausted all the routine treatments, a trial of therapy with compassionate use protocol was the last-ditch option, discussed with the patient and obtained signed consent form and IRB approval. Based on the genomic profile, we speculated that the PI3K-Akt axis could play an important role in the growth of the lung cancer and potentially the CCRCC. The ethics committee of the Affiliated Zhongshan Hospital of Dalian University approved the study (The Institutional Review Board approval number is 2016102). A combination of PIK3CA inhibitor BKM120 (80mg PO QD) ${ }^{5}$ and AKT1 inhibitor MK2206 (45mg PO QOD $)^{5}$ on a 28-day cycle was initiated starting from February, 2015. The clinicians determined the doses in reference to the available clinical trials NCT01294306, BATTLE-2, NCT01911325, and BASALT-1 in the year of 2015. The combination of BKM120 and MK2206 was expected to suppress growth of both CCRCC and lung SQCC. For CCRCC, Sorafenib was discontinued. For prostate adenocarcinoma, PSA level was continued to be monthly monitored, and subcutaneous Goserelin was administered whenever an increase of PSA was observed.

After beginning of the targeted therapy, the patient was monthly followed-up. At revisits after 4 months and 9 months in June and December 2015, chest CT showed a shrinkage of lung tumor size by $40 \%$ and $80 \%$ (Figure 2E and F), indicating that the targeted therapy had a remarkable therapeutical effect on the lung SQCC with continuous partial-response (PR). Meanwhile, no sign of visceral metastasis was found by abdominal CT scan (Figure $2 \mathrm{G}$ and $\mathrm{H}$ ), suggesting that $\mathrm{CCRCC}$ and prostate adenocarcinoma were under effective control. The patient maintained the described therapy and the tumor remained responsive until death in March 2016. The main adverse effects were mild fatigue, nausea, and repetitive watery diarrhea and resultant hypokalemia which were managed 


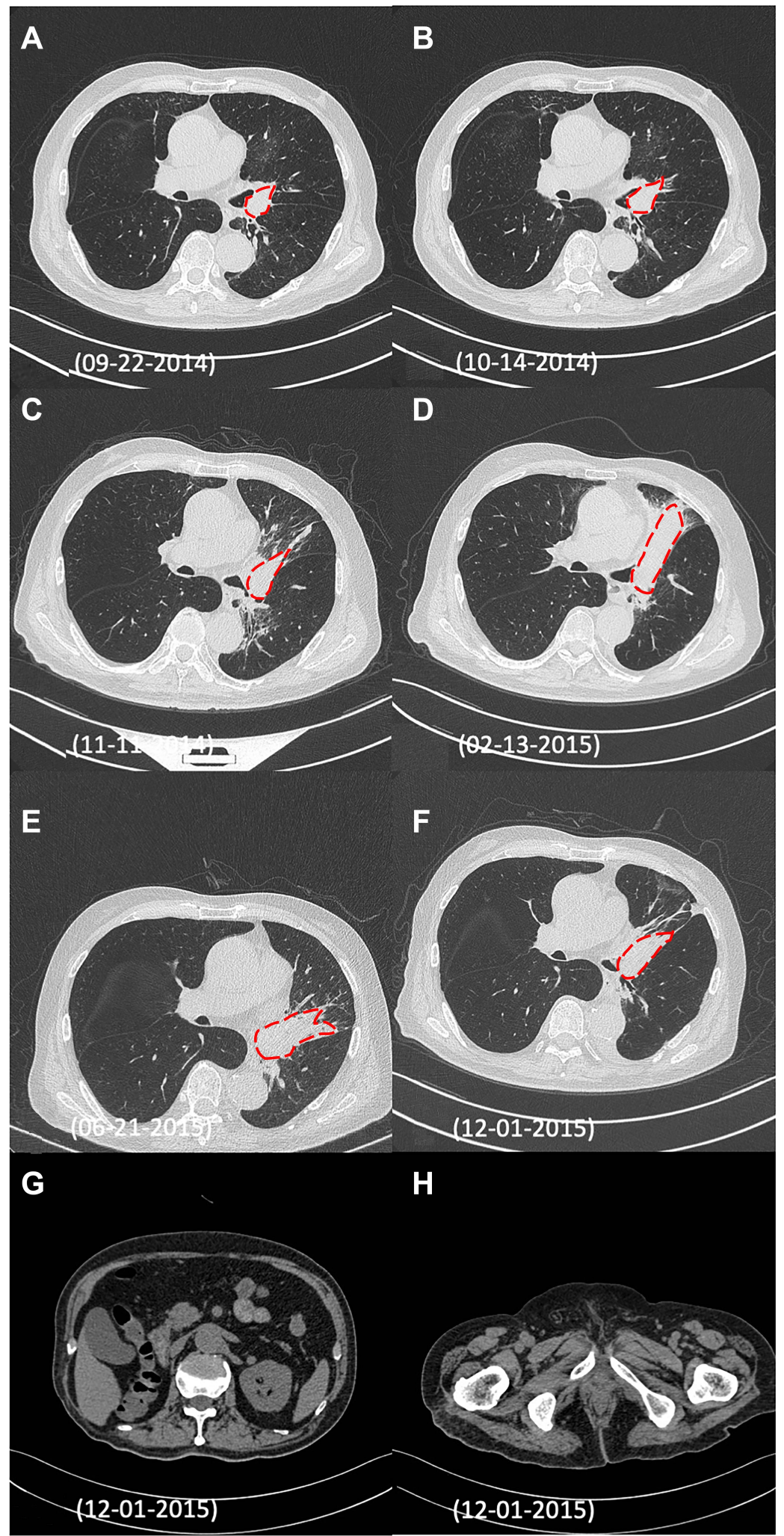

Figure $2 \mathrm{CT}$ images reflect the dynamic changes of the patient's responses to the treatment plans. (A) Size of lung SQCC ( $1.7 \times 1.7 \mathrm{~cm}$, red dotted line) soon after the initial diagnosis by fiberoptic bronchoscopy; (B) Radiation pneumonitis and unchanged tumor size ( $1.7 \times 1.7 \mathrm{~cm}$, red dotted line) after radiotherapy; C) Radiation pneumonitis and increased tumor size $(2.1 \times 2.8 \mathrm{~cm}$, red dotted line) after radiotherapy; (D) Increased tumor size $(3.1 \times 9.4 \mathrm{~cm}$, red dotted line) after platinum-based chemotherapy; (E) Reduced tumor size $(3.5 \times 6.3 \mathrm{~cm}$, red dotted line) 4 months after targeted therapy; (F) Further reduced tumor size $(2.47 \times 2.47 \mathrm{~cm}$, red dotted line) 9 months after targeted therapy; (G and $\mathbf{H}$ ) Follow-up images of CCRC and prostate adenocarcinoma in 2015 showed no sign of tumor recurrence. 
Table I Mutation Profiles of the Lung Squamous Cell Carcinoma, Renal Clear Cell Carcinoma, Prostate Adenocarcinoma and Metastatic Bone Tumor Using Targeted Exome Sequencing

\begin{tabular}{|c|c|c|c|c|}
\hline $\begin{array}{l}\text { Significantly } \\
\text { Mutated Gene }\end{array}$ & Lung SQCC & Metastatic Bone Tumor & $\begin{array}{c}\text { Prostate } \\
\text { Adenocarcinoma }\end{array}$ & CCRCC \\
\hline VHL & $\begin{array}{c}\text { c.43IA>G|p.Tyr I44Cys } \\
(\text { VCV000223232) }\end{array}$ & $\begin{array}{c}\text { c.554A > T |p.Tyr I85Phe } \\
(\text { COSV56542976) }\end{array}$ & & $\begin{array}{c}\text { c.554A > T |p.Tyr I85Phe } \\
(\text { COSV56542976) }\end{array}$ \\
\hline PIK3CA & $\begin{array}{c}\text { c.3।42C>T|p.His I048Tyr } \\
(\text { COSM249875) }\end{array}$ & & & \\
\hline EGFR & $\begin{array}{c}\text { c.2387G>A|p.Gly796Asp } \\
(\text { COSV5I776I9I) }\end{array}$ & & & \\
\hline BRAF & $\begin{array}{l}\text { c. } 18 \text { IIG>A|p.Trp604* } \\
(\text { COSV56I99774) }\end{array}$ & & & \\
\hline FGFR2 & & $\begin{array}{c}\text { c.542A>G|p.Lys I I I Arg } \\
(\text { COSV60642289) }\end{array}$ & & $\begin{array}{c}\text { c.542A>G|p.Lys I 8I Arg } \\
(\text { COSV60642289) }\end{array}$ \\
\hline HRAS & $\begin{array}{l}\text { c.|30G>A|p.Val44Met } \\
(\text { COSVI043729I0) }\end{array}$ & & & \\
\hline AKTI & $\begin{array}{c}\text { c.68G>A|p.Arg23Gln } \\
(\text { COSM3770600) }\end{array}$ & & & $\begin{array}{l}\text { c.49G>A| p.Glu I 7Lys } \\
(\text { COSM33765) }\end{array}$ \\
\hline $\mathrm{CDHI}$ & $\begin{array}{c}\text { c. } 1298 \text { A>G|p.Asp433Gly } \\
(\text { COSV99219933) }\end{array}$ & & & \\
\hline TP53 & $\begin{array}{c}\text { c.838A > T|p.Arg280* } \\
(\text { COSV52708728) }\end{array}$ & $\begin{array}{c}\text { c.565G>C|p.Ala | 89Pro } \\
(\text { COSV52875466) }\end{array}$ & & $\begin{array}{c}\text { c.565G >Clp.Ala | 89Pro } \\
(\text { COSV52875466) }\end{array}$ \\
\hline
\end{tabular}

Note: *Indicates deletion.

Abbreviations: SQCC, squamous cell carcinoma; CCRCC, clear cell renal carcinoma.

by dioctahedral smectite, PO/IV fluids, and electrolyte supplementation. The glucose level, renal, and liver function test were all monitored and no life-threatening complications were observed. However, the patient developed gradual collapse of the left lung (Figure 2C-F) due to occlusion on the hilum, respiratory function deterioration, and eventually died of a complication of pulmonary infection and cardiopulmonary failure in March 2016. Our findings demonstrate a good example of beneficial application of targeted therapy combining PI3K and AKT inhibitors to treat refractory and complicated cases of MPC/ NSCLC and may inspire more investigations in this field.

\section{Discussion and Conclusion}

The reports of MPC cases have been accumulating over the past few decades. ${ }^{1,2}$ However, the treatment may frequently become tricky when one or more complicating factors could superimpose upon each other. In the present case, the patient had an obstinate lung SQCC together with renal and prostate tumors. SQCC of the lung is a subtype of non-small cell lung cancer (NSCLC) which prognosis remains poor to date. ${ }^{5}$ Frequently, lung SQCC has a central airway location impossible for radical lobectomy, its treatment heavily relies on the platinum-based chemotherapy. ${ }^{5}$ In our case, the patient had no surgical indications, radiotherapy and chemotherapy failed soon, and a trial of compassionate use protocol considered genome-based targeted therapy as the last option, although the genomic profile and validated targets for lung SQCC remained comparatively much underdeveloped than those for the lung adenocarcinoma. Notably, by the time of early 2015, the immunotherapy which is one of the most recently advanced therapeutical approaches for lung SQCC remained under evaluation.

Genomic studies of SQCC have been a hot topic for NSCLC research looking for some novel and more effective therapeutical targets. ${ }^{5}$ According to the Cancer Genome Atlas (TCGA), the hot- spot mutations reported in SQCC are TP53, CDKN2A, PTEN, PIK3CA, KEAP1, MLL2, AKT1, NFE2L2, NOTCH1, and RB1. ${ }^{5,6}$ Our findings are consistent with the TCGA reports, with PIK3CA and AKT1 mutations being identified and employed as 
drug targets, along with the other mutations on EGFR, BRAF, CDH1, HRAS, and TP53 (Table 1). A combination of PIK3CA inhibitor Buparlisib (BKM120) and AKT inhibitor MK2206 was used to treat the lung SQCC of our patient presented here. BKM120 and MK2206, as newer targeted drugs inhibiting the PI3KAkt signaling pathway, are currently on clinical trials for NSCLC ${ }^{5-13}$ Aberrant presence of PIK3C isoforms have been reported in lung SQCC, with mutation frequency of $3 \sim 6 \%$ and over-expression frequency of $16 \sim 40 \%{ }^{5,6} \mathrm{PI} 3 \mathrm{~K}-$ Akt/EGFR-MAPK pathways have been suggested central to the carcinogenesis and tumor growth for NSCLC. ${ }^{5}$ However, two clinical trials of BKM120 on lung SQCC have failed. The phase-II BASALT-1 study failed to show increased PFS in receiving BKM120. ${ }^{10}$ The NCT01911325 Phase II trial showed only a marginal antitumor activity by BKM120 plus docetaxel. ${ }^{11}$ Whether an alternative combination of BKM120 with the other medications would have better efficacy remains to be determined. Similarly, in a study of Erlotinib-resistant NSCLC, the AKT inhibitor MK2206 seemed unable to potentiate the efficacy of Erlotinib. ${ }^{12}$ The BATTLE-2 trial aimed to compare the efficacy of MK2206 in NSCLC with mutant or wild type KRAS and the study remained ongoing. ${ }^{13}$ Currently there have been no studies using combined MK2206 and BKM120 in NSCLC/SQCC treatment. Our case demonstrated a good combination of BKM120 and MK2206 to combat lung SQCC and possibly CCRCC with actionable AKT1 mutation, which is worth further clinical investigations.

Our case presented another primary cancer CCRCC. According to an analysis of the SEER database 1975-2001, MPC involving both lung and kidney accounts for $5 \%$ of all MPC cases. ${ }^{1}$ Smoking is the major risk factor for CCRCC and gene mutation including VHL gene and genes on the PI3K-Akt-mTOR axis is another known risk factor. ${ }^{14}$ The targeted therapy of CCRCC included antiangiogenic TKIs targeting the VEGF signaling, mTOR inhibitors, and immunotherapies. ${ }^{14}$ While clinical interest in PI3K-Akt inhibitors is rising rapidly, certain phase I/II trials demonstrated a great potential of BKM120 and MK2206 in CCRCC treatment. A Phase I study of BKM120 plus bevacizumab showed partial response in 13\% metastatic CCRCC patients, with increased efficacy in those harboring activating PI3K mutations. ${ }^{15}$ Another phase II study in VEGF inhibitor-refractory patients showed an efficacy similar to the mTOR inhibitor Everolimus. ${ }^{16}$ Although in our case, the patient's renal cancer could have been cured by Sorafenib and no further bone metastasis had been reported for years, our decision to use BKM120 combined with MK2206 may exert certain protective function against the CCRCC in overt stage.

In a summary, in the present study we reported a rare case of multiple primary cancers involving obstinate lung squamous cell carcinoma, clear cell renal cell carcinoma, and prostate adenocarcinoma. Genome-guided targeted therapy identified mutations aberrantly affecting the PI3KAkt pathway across different tumors and a combination of PI3K inhibitor BKM120 and AKT inhibitor MK2206 achieved good efficacy prolonging the patient's survival for more than a year. Our findings demonstrated a good example of precision medicine in the treatment of rare and difficult clinical scenarios of multiple primary cancers.

\section{Data Sharing Statement}

All data generated or analyzed during this study are included in this published article and its supplementary information file.

\section{Ethics Approval and Consent to Participate}

Written informed consent was obtained from the patient in accordance with the Declaration of Helsinki for the purpose of publication of the present case report and any relevant images.

\section{Consent for Publication}

Written informed consent was obtained from the patient for publication of the case report and the accompanying images.

\section{Acknowledgments}

We would like to thank Dr. Tim Lilburn for critical reading of the manuscript.

\section{Funding}

This work is supported by an Innovation Grant for Science and Technology from the Dalian Government to YD for design and development of targeted sequencing panels and funding from the Affiliated Zhongshan Hospital of Dalian University to RYW for conducting the experiment.

\section{Disclosure}

The authors declare that they have no competing interests. 


\section{References}

1. Mariotto AB, Rowland JH, Ries LA, Scoppa S, Feuer EJ. Multiple cancer prevalence: a growing challenge in long-term survivorship. Cancer Epidemiol Biomarkers Prev. 2007;16(3):566-571. doi:10.1158/1055-9965.EPI-06-0782

2. Hayat MJ, Howlader N, Reichman ME, Edwards BK. Cancer statistics, trends, and multiple primary cancer analyses from the Surveillance, Epidemiology, and End Results (SEER) Program. Oncologist. 2007;12(1):20-37. doi:10.1634/theoncologist.12$1-20$

3. Fujita S, Masago K, Takeshita J, et al. Multiple primary malignancies in patients with non-small cell lung cancer. Intern Med. 2015;54 (3):325-331. doi:10.2169/internalmedicine.54.2921

4. Afghahi A, Sledge GW. Targeted Therapy for Cancer in the Genomic Era. Cancer J. 2015;21(4):294-298. doi:10.1097/PPO.0000 000000000135

5. Filipits M. New developments in the treatment of squamous cell lung cancer. CurrOpinOncol. 2014;26(2):152-158.

6. The Cancer Genome Atlas Research Network. Comprehensive genomic and characterization of squamous cell lung cancers. Nature. 2012;489:519-525. doi:10.1038/nature11404

7. Monnet I, Audigier-Valette C, Girard N, et al. Real-life effectiveness of Erlotinib as second-line treatment of stage IIIB/IV squamous non-small cell lung cancer: results of the PEPiTA observational study. Lung Cancer. 2016;98:84-90. doi:10.1016/j.lungcan.2 016.05.016

8. Peters S, Stahel RA, Dafni U, et al. EMPHASIS-lung Collaborative Group.Randomized Phase III Trial of Erlotinib versus Docetaxel in Patients with Advanced Squamous Cell Non-Small Cell Lung Cancer Failing First-Line Platinum-Based Doublet Chemotherapy Stratified by VeriStrat Good versus VeriStrat Poor. The European Thoracic Oncology Platform (ETOP) EMPHASIS-lung Trial. $J$ Thorac Oncol. 2017;12(4):752-762.
9. Paik PK, Varghese AM, Sima CS, et al. Response to Erlotinib in patients with EGFR mutant advanced non-small cell lung cancers with a squamous or squamous-like component. Mol Cancer Ther. 2012;11(11):2535-2540. doi:10.1158/1535-7163.MCT-12-0163

10. Vansteenkiste JF, Canon JL, De Braud F, et al. Safety and Efficacy of Buparlisib (BKM120) in Patients with PI3K Pathway-Activated Non-Small Cell Lung Cancer: results from the Phase II BASALT-1 Study. $\quad J$ Thorac Oncol. 2015;10(9):1319-1327. doi:10.1097/ JTO.0000000000000607

11. Adjei AA, Bennouna J, Leighl NB, et al. Safety and efficacy of buparlisib (BKM120) and chemotherapy in advanced, squamous non-small cell lung cancer (sqNSCLC): Results from the phase $\mathrm{Ib} /$ II BASALT-2 and BASALT-3 studies. J Clin Oncol. 2016;34 (15_suppl.):e20522. doi:10.1200/JCO.2016.34.15_suppl.e20522

12. Lara PN, Longmate J, Mack PC, et al. Phase II Study of the AKT Inhibitor MK-2206 plus Erlotinib in Patients with Advanced Non-Small Cell Lung Cancer Who Previously Progressed on Erlotinib. Clin Cancer Res. 2015;21(19):4321-4326. doi:10.1158/ 1078-0432.CCR-14-3281

13. Papadimitrakopoulou V, Lee JJ, Wistuba II, et al. The BATTLE-2 Study: a Biomarker-IntegratedTargeted Therapy Study in Previously Treated Patients With Advanced Non-Small-Cell Lung Cancer. J Clin Oncol. 2016;34(30):3638-3647. doi:10.1200/JCO.2015.66.0084

14. Shingarev R, Jaimes EA. Renal cell carcinoma: new insights and challenges for a clinician scientist. Am J Physiol Renal Physiol. 2017;313(2):F145-F154. doi:10.1152/ajprenal.00480.2016

15. McKay RR, De Velasco G, Werner L, et al. A Phase 1 study of Buparlisib and bevacizumab in patients with metastatic renal cell carcinoma progressing on vascular endothelial growth factor-targeted therapies. Cancer. 2016;122(15):2389-2398. doi:10.1002/cncr.30056

16. Jonasch E, Hasanov E, Corn PG, et al. A randomized Phase 2 study of MK-2206 versus everolimus in refractory renal cell carcinoma. Ann Oncol. 2017;28(4):804-808. doi:10.1093/annonc/mdw676
OncoTargets and Therapy

\section{Publish your work in this journal}

OncoTargets and Therapy is an international, peer-reviewed, open access journal focusing on the pathological basis of all cancers, potential targets for therapy and treatment protocols employed to improve the management of cancer patients. The journal also focuses on the impact of management programs and new therapeutic

Submit your manuscript here: https://www.dovepress.com/oncotargets-and-therapy-journal agents and protocols on patient perspectives such as quality of life, adherence and satisfaction. The manuscript management system is completely online and includes a very quick and fair peer-review system, which is all easy to use. Visit http://www.dovepress.com/ testimonials.php to read real quotes from published authors. 